\title{
Complex wound closure by plastic surgery following resection of spinal neoplasms minimizes postoperative wound complications in high-risk patients
}

\author{
Owen P. Leary, ScB, ${ }^{1,3}$ David D. Liu, ScB, ${ }^{1}$ Michael K. Boyajian, BA, ${ }^{2}$ Sohail Syed, MD, ${ }^{1,3}$ \\ Joaquin Q. Camara-Quintana, MD, ${ }^{1,3}$ Tianyi Niu, MD, ${ }^{1,3}$ Konstantina A. Svokos, DO, MS, ${ }^{1,3}$ \\ Joseph Crozier, MA, ${ }^{2,3}$ Adetokunbo A. Oyelese, MD, PhD, ${ }^{1,3}$ Paul Y. Liu, MD, ${ }^{2,3}$ Albert S. Woo, MD, ${ }^{2,3}$ \\ Ziya L. Gokaslan, MD, ${ }^{1,3}$ and Jared S. Fridley, MD ${ }^{1,3}$
}

Departments of ${ }^{1}$ Neurosurgery and ${ }^{2}$ Plastic Surgery, Warren Alpert Medical School of Brown University; and ${ }^{3}$ Lifespan Health System/Rhode Island Hospital, Providence, Rhode Island

OBJECTIVE Wound breakdown and infection are common postoperative complications following resection of spinal neoplasms. Accordingly, it has become common practice at some centers for plastic surgeons to assist with closure of large posterior defects after spine tumor resection. In this study, the authors tested the hypothesis that plastic surgery closure of complex spinal defects improves wound outcomes following resection of spinal neoplastic disease.

METHODS Electronic medical records of consecutive patients who underwent resection of a spinal neoplasm between June 2015 and January 2019 were retrospectively reviewed. Patients were separated into two subpopulations based on whether the surgical wound was closed by plastic surgery or neurosurgery. Patient demographics, preoperative risk factors, surgical details, and postoperative outcomes were collected in a central database and summarized using descriptive statistics. Outcomes of interest included rates of wound complication, reoperation, and mortality. Known preoperative risk factors for wound complication in spinal oncology were identified based on literature review and grouped categorically. The presence of each category of risk factors was then compared between groups. Univariate and multivariate linear regressions were applied to define associations between individual risk factors and wound complications.

RESULTS One hundred six patients met inclusion criteria, including 60 wounds primarily closed by plastic surgery and 46 by neurosurgery. The plastic surgery population included more patients with systemic metastases ( $58 \%$ vs $37 \%, p=$ $0.029)$, prior radiation ( $53 \%$ vs $17 \%, p<0.001)$, prior chemotherapy $(37 \%$ vs $15 \%, p=0.014)$, and sacral region tumors ( $25 \%$ vs $7 \%, p=0.012$ ), and more patients who underwent procedures requiring larger incisions $(7.2 \pm 3.6$ vs $4.5 \pm 2.6$ levels, $p<0.001$ ), prolonged operative time (413 \pm 161 vs $301 \pm 181$ minutes, $p=0.001)$, and greater blood loss (906 \pm 1106 vs $283 \pm 373 \mathrm{ml}, p<0.001)$. The average number of risk factor categories present was significantly greater in the plastic surgery group $(2.57$ vs $1.74, p<0.001)$. Despite the higher relative risk, the plastic surgery group did not experience a significantly higher rate of wound complication ( $28 \%$ vs $17 \%, p=0.145)$, reoperation $(17 \%$ vs $9 \%, p=0.234)$, or all-cause mortality ( $30 \%$ vs $13 \%, p=0.076)$. One patient died from wound-related complications in each group $(p=$ 0.851). Regression analyses identified diabetes, multilevel instrumentation, and BMI as the factors associated with the greatest wound complications.

CONCLUSIONS Involving plastic surgery in the closure of spinal wounds after resection of neoplasms may ameliorate expected increases in wound complications among higher-risk patients.

https://thejns.org/doi/abs/10.3171/2019.12.SPINE191238

KEYWORDS spinal oncology; wound complications; complex spine surgery; spinal fixation; risk stratification

ABBREVIATIONS ASA = American Society of Anesthesiologists; EMR = electronic medical record; $S S I$ = surgical site infection .

SUBMITTED October 16, 2019. ACCEPTED December 31, 2019.

INCLUDE WHEN CITING Published online February 28, 2020; DOI: 10.3171/2019.12.SPINE191238. 
$\mathrm{P}$ ATIENTS undergoing resection of spinal neoplasms are typically considered to be at higher risk for postoperative wound complications than other spine surgery populations. Common contributors to increased risk of surgical site infection (SSI), wound breakdown, and need for reoperation in the setting of neoplastic spinal disease include larger posterior incision size, more significant disruption of bony and paraspinous tissue, and higher prevalence of medical pre- and postoperative risk factors. ${ }^{24,26,28}$ Many such risk factors (e.g., prior cancer treatment, smoking history, diabetes, and chronic steroid usage) are unavoidable or uncontrollable at the time of urgent resection. As a result, the spine surgeon's armamentarium for optimizing wound outcomes for these patients is often limited to intra- and postoperative management of the wound itself.

While simple layer-by-layer tissue reapproximation for primary closure of posterior defects remains the standard of care for most open spine surgery cases, mobilization of paraspinal muscle flaps for wound coverage during closure is an approach that has long been utilized in revision surgeries for management of postoperative wound dehiscence

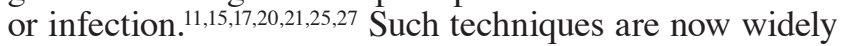
utilized by plastic surgeons for repair, debridement, and salvage of infected or deteriorating back wounds. Indeed, these methods were founded on extensive pathobiology literature demonstrating that mobilized muscle flaps are more resistant to bacterial infection than simple layered closure at a wound site. ${ }^{5,12,22}$ More recently, other groups have published small retrospective reviews and singlesurgeon case series demonstrating the safety and efficacy of prophylactic soft-tissue reconstruction with musculocutaneous flaps. These studies advocate muscle flap reconstruction as a primary closure technique for preventing infection in complex spine cases rather than exclusively as a secondary approach for reapproximating a wound after a complication has already occurred. ${ }^{6-9,13,14}$

In June of 2015, our neurosurgery department began to increasingly involve plastic surgery in the primary closure of large spinal wounds believed to be at risk for complication after complex spine tumor resection. We now present the resultant single-institution, multisurgeon, retrospective case-control study comparing wound-related complication rates in consecutive patients who did or did not have primary plastic surgery closure of their complex posterior defect after resection of neoplastic disease. Furthermore, we stratified relative risk of poor wound outcome in the spinal oncology population in terms of number of risk factor categories present preoperatively and performed regression analyses to identify positive associations between individual known risk factors and various observed wound complications. These data motivate future studies focused on building a novel wound complication risk scoring system specific to this population.

\section{Methods}

\section{Patient Population}

We reviewed the electronic medical records (EMRs) of all patients who underwent spinal surgery for resection of a suspected tumor within the Department of Neurosurgery at Rhode Island Hospital between June 1, 2015, and January 31, 2019. Patients were identified using a comprehensive list of all spine surgeries performed in the department over this period and by reviewing all procedure descriptions to identify language describing resection of a tumor or spinal mass. All records were reviewed to confirm the presence of tumor or suspected spinal mass prior to inclusion determination. There were no exclusion criteria based on availability of follow-up data. This study was approved by the Rhode Island Hospital IRB as a retrospective chart review of the EMR, which authorized exemption from informed consent requirements.

\section{Surgical Technique}

All patients who underwent wound closure with plastic surgery received immediate coverage with local muscle flaps. Flap options included paraspinous, latissimus dorsi, trapezius, and thoracolumbar flaps. The choice of flap depended on the location of the spinal wound. A typical back wound triaged for closure by plastic surgery after resection of a spinal tumor at our center is depicted in Fig. 1. Conversely, patients who underwent wound closure with neurosurgery alone were more likely to undergo simple layered reapproximation of muscle, fascia, and cutaneous tissue.

\section{Data Collection}

At least one of four independent chart reviewers studied all clinic and operative notes available in the EMR for included subjects. Demographic, baseline, and preoperative risk factor details were obtained from history and physical examination, and preoperative office visit notes. Surgical details were obtained from operative notes, and tumor diagnoses were confirmed from an uploaded pathology report in each case. References to postoperative wound complications in postoperative hospital, emergency department, or office visit notes were used to document these outcomes up to the most recent follow-up visit at the time of this submission. Outcomes of interest included surgical site infection (SSI), skin necrosis, wound dehiscence, hematoma, seroma, CSF leak, reoperation, and mortality. The first six of these were also grouped into a more expansive ninth outcome variable ("any wound complication"). As our institution is the leading comprehensive subspecialty center in our region, we expect that the vast majority of postoperative emergency and surgical care for these subjects was sought at our center and was therefore documented in our EMR.

\section{Statistical Analyses}

Descriptive analyses were conducted by grouping patients as a single cohort, and by separating them into two subpopulations defined by whether or not the patient's surgical wound was closed by a plastic surgeon or a neurosurgeon. Regression analyses of association between risk factors and individual outcomes were performed on the full patient cohort. Descriptive statistics were conducted using Microsoft Excel (version 16.16.4, Microsoft Corp.), and regression analyses performed in the StatsModel module of Python (version 3.6.5). Continuous variable data are re- 

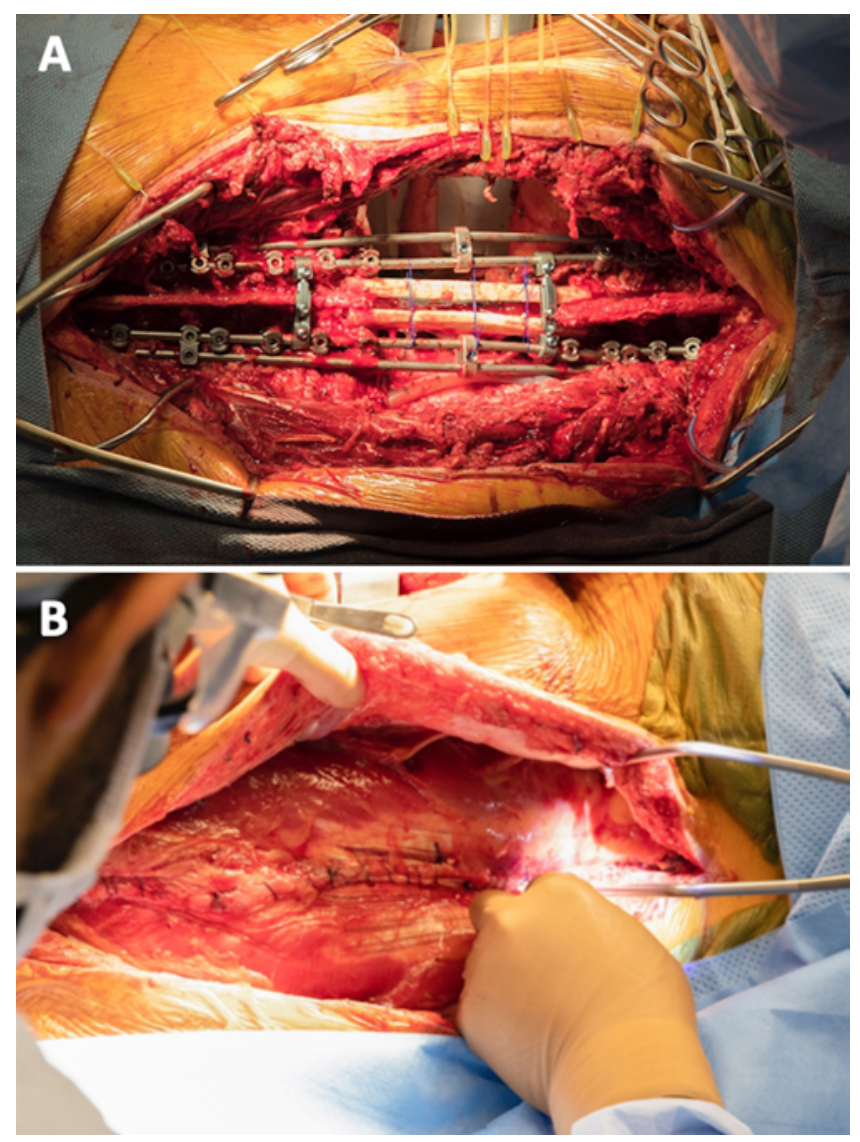

FIG. 1. Exemplary case of complex wound closure by plastic surgery following resection of a thoracic chordoma and placement of bilateral posterior hardware and fibular allografts. A: Posterior wound after hardware placement. B: Sutured subcutaneous paraspinous muscle flaps. Figure is available in color online only.

ported in terms of averages and standard deviations, while binary variable data are reported in terms of percentage of patients.

\section{Risk Stratification}

Spinal oncology patients often present with multiple preoperative risk factors for wound complication. However, after review of the available literature, it was determined that there is currently no adequate risk scoring system for spinal oncology patients that accounts for multisource wound complication risk and stratifies patients accordingly. Our literature review did identify known risk factors for the wound outcomes of interest. . $^{1,2,6,9,13,19,24,26,28,29}$ We then grouped preoperative risk factors categorically (Table 1). Three of the four categories, including medical history factors (smoking, diabetes, hypertension, obesity), chronic medications of risk (anticoagulation, steroids), and prior cancer treatments (chemotherapy, radiation, spine surgery), comprise known preoperative medical risk factors. The fourth includes procedure planning factors, comprising the number of spinal levels to be incised and anticipated instrumentation. By identifying the presence of these risk factors for each patient, we were able to quantify the number of risk factor categories present (out of four
TABLE 1. Key factors associated with wound complication risk in spinal oncology patients

\begin{tabular}{c}
\hline Risk Factor Category \\
\hline Past medical history factors \\
\hline Smoking history? \\
\hline Treatment for diabetes? \\
\hline History of hypertension? \\
\hline BMl $>30 \mathrm{~kg} / \mathrm{m}^{2} ?$ \\
\hline Medication factors \\
\hline Chronic blood thinners? \\
\hline Chronic steroids? \\
\hline Prior treatment factors \\
\hline Prior chemotherapy? \\
\hline Prior radiation? \\
\hline Prior spine surgery? \\
\hline Size of posterior spine wound \\
\hline No. of levels incised $>5 ?$ \\
\hline No. of levels instrumented $>4 ?$ \\
\hline
\end{tabular}

Risk factors were grouped into four key categories easily assessable in the preoperative setting, so that the number of risk factor categories present could also be assessed as a composite risk metric.

key categories) and used this composite metric as a means of stratifying preoperative risk for intergroup comparison. We also conducted post hoc univariate and multivariate regression analyses to detect associations of individual risk factors, as well as the composite number of risk categories, with each of the negative wound outcomes we documented in the study.

\section{Results \\ Patient Characteristics}

A total of 106 patients with spine tumors were included in the study, including 60 patients who underwent primary wound closure with plastic surgery and 46 who underwent closure with neurosurgery. Demographic, medical history, and tumor diagnosis data are presented in Table 2. The two groups were not significantly different in terms of age (mean $58 \pm 15.9$ vs $57 \pm 17.6$ years, $\mathrm{p}=0.751)$, sex $(57 \%$ vs $43 \%$ male, $p=0.182$ ), or BMI (mean $28.1 \pm 5.4$ vs $28.5 \pm$ $6.1 \mathrm{~kg} / \mathrm{m}^{2}, \mathrm{p}=0.713$ ). Regarding past medical history data, the groups differed significantly in terms of past cancer treatments, including prior chemotherapy $(37 \%$ in plastic surgery group vs $15 \%$ in neurosurgery group, $p=0.014$ ) and prior radiation $(53 \%$ vs $17 \%, \mathrm{p}<0.001)$. Patient groups were not significantly different in terms of American Society of Anesthesiologists (ASA) classification (mean 2.8 \pm 0.6 vs $2.7 \pm 0.8, \mathrm{p}=0.245$ ).

Overall, although the data set encompassed a wide variety of primary and metastatic spine tumor subtypes, the groups were significantly different in terms of diagnostic profile. While $58 \%$ of the patients in the plastic surgery group underwent spine surgery to treat metastatic disease, as opposed to a primary spine tumor, only $37 \%$ of the patients in the neurosurgery group did $(\mathrm{p}=0.029)$. Furthermore, and consistent with the initial recommendation, the 
TABLE 2. Demographic, physical, medical history, and tumor characteristic details for the full patient cohort, as well as for complex (plastic surgery) and simple (neurosurgery) wound closure subpopulations

\begin{tabular}{|c|c|c|c|c|}
\hline Patient Characteristics & Total Cohort $(n=106)$ & Complex Closure Group $(n=60)$ & Simple Closure Group $(n=46)$ & $\mathrm{p}$ Value \\
\hline \multicolumn{5}{|l|}{ Demographic \& physical details } \\
\hline Male, n (\%) & $57(54)$ & $34(57)$ & $20(43)$ & 0.182 \\
\hline Mean age $\pm S D$, yrs & $57 \pm 16.7$ & $58 \pm 15.9$ & $57 \pm 17.6$ & 0.751 \\
\hline Mean height $\pm S D$, inches & $66.3 \pm 4.1$ & $66.7 \pm 3.9$ & $65.8 \pm 4.2$ & 0.289 \\
\hline Mean weight $\pm S D$, lbs & $176.6 \pm 39.4$ & $175.8 \pm 42.1$ & $177.3 \pm 37.5$ & 0.855 \\
\hline Mean $\mathrm{BMI} \pm \mathrm{SD}, \mathrm{kg} / \mathrm{m}^{2}$ & $28.2 \pm 5.69$ & $28.1 \pm 5.4$ & $28.5 \pm 6.1$ & 0.713 \\
\hline Mean ASA physical status \pm SD & $2.7 \pm 0.7$ & $2.8 \pm 0.6$ & $2.7 \pm 0.8$ & 0.245 \\
\hline \multicolumn{5}{|l|}{ Past medical history, $\mathrm{n}(\%)$} \\
\hline Smoking history & $58(55)$ & $35(58)$ & $23(50)$ & 0.398 \\
\hline Diabetes & $13(12)$ & $5(8)$ & $8(17)$ & 0.162 \\
\hline Hypertension & $42(40)$ & $22(37)$ & $20(43)$ & 0.482 \\
\hline CVA/stroke & $8(8)$ & $3(5)$ & $5(11)$ & 0.261 \\
\hline Coagulopathy/chronic AC & $23(22)$ & $13(22)$ & $10(22)$ & 0.992 \\
\hline Chronic steroid usage & $17(16)$ & $10(17)$ & $7(15)$ & 0.842 \\
\hline Prior chemotherapy & $29(27)$ & $22(37)$ & $7(15)$ & 0.014 \\
\hline Prior radiation & $40(38)$ & $32(53)$ & $8(17)$ & $<0.001$ \\
\hline Prior spine surgery & $19(18)$ & $14(23)$ & $5(11)$ & 0.099 \\
\hline \multicolumn{5}{|l|}{ Tumor type, n (\%) } \\
\hline Primary spinal tumor & $54(51)$ & $25(42)$ & $29(63)$ & 0.029 \\
\hline Spinal metastases & $52(49)$ & $35(58)$ & $17(37)$ & 0.029 \\
\hline \multicolumn{5}{|l|}{ Tumor diagnosis, $\mathrm{n}(\%)$} \\
\hline Schwannoma & $17(16)$ & $4(7)$ & $13(28)$ & 0.002 \\
\hline Meningioma & $5(5)$ & $0(0)$ & $5(11)$ & 0.009 \\
\hline Ependymoma & $8(8)$ & $1(2)$ & $7(15)$ & 0.009 \\
\hline Chordoma & $13(12)$ & $12(20)$ & $1(2)$ & 0.005 \\
\hline Spinal cord glioma & $1(1)$ & $0(0)$ & $1(2)$ & 0.255 \\
\hline Lung metastasis & $14(13)$ & $10(17)$ & $4(9)$ & 0.234 \\
\hline Prostate metastasis & $9(8)$ & $3(5)$ & $6(13)$ & 0.144 \\
\hline Breast metastasis & $7(7)$ & $4(7)$ & $3(7)$ & 0.977 \\
\hline Liver metastasis & $2(2)$ & $1(2)$ & $1(2)$ & 0.851 \\
\hline Thyroid metastasis & $2(2)$ & $2(3)$ & $0(0)$ & 0.215 \\
\hline Lymphoma & $3(3)$ & $1(2)$ & $2(4)$ & 0.414 \\
\hline Renal cell carcinoma metastasis & $9(8)$ & $9(15)$ & $0(0)$ & 0.006 \\
\hline Other primary tumor* & $10(9)$ & $8(13)$ & $2(4)$ & 0.119 \\
\hline Other metastatic tumor $†$ & $6(6)$ & $5(8)$ & $1(2)$ & 0.177 \\
\hline \multicolumn{5}{|l|}{ Target lesion location, n (\%)‡ } \\
\hline Cervical (C1-7) & $15(14)$ & $9(15)$ & $6(13)$ & 0.777 \\
\hline Thoracic (T1-12) & $57(54)$ & $28(47)$ & $29(63)$ & 0.095 \\
\hline Lumbar (L1-5) & $27(25)$ & $15(25)$ & $12(26)$ & 0.900 \\
\hline Sacral/coccygeal & $18(17)$ & $15(25)$ & $3(7)$ & 0.012 \\
\hline \multicolumn{5}{|c|}{$\begin{array}{l}\text { AC = anticoagulation; CVA = cerebrovascular accident. } \\
\text { Continuous variables reported as averages and standard deviations. The } p \text { values represent results of unpaired t-tests of difference between the two subpopulations } \\
\text { with respect to the variable indicated (significance presumed if } p<0.05 \text {, and high significance presumed if } p<0.001) \text {. Boldface type indicates statistical significance. } \\
\text { * Other primary tumors included malignant peripheral nerve sheath tumor }(n=1) \text {, calcified synovial cyst }(n=1) \text {, osteoid osteoma }(n=1) \text {, solitary fibrous tumor }(n=1) \text {, } \\
\text { hemangioma }(n=1) \text {, hemangioendothelioma }(n=1) \text {, giant cell tumor }(n=1) \text {, chondroblastoma }(n=1) \text {, lipoma }(n=1) \text {, and fibrous dysplasia }(n=1) \text {. } \\
\text { † Other metastatic tumors included melanoma }(n=1) \text {, high-grade liposarcoma }(n=1) \text {, endometrial cancer }(n=1) \text {, oropharyngeal cancer }(n=1) \text {, esophageal cancer }(n= \\
\text { 1), and bile duct cancer }(n=1) \text {. } \\
\text { † Some target lesions span two spinal regions; thus, percentages sum to }>100 \% \text {. }\end{array}$} \\
\hline
\end{tabular}


group of patients who underwent complex plastic closure had larger lesions more likely to exhibit aggressive pathology. While the neurosurgery closure group included significantly more schwannomas $(28 \%$ of tumors vs $7 \%$, $\mathrm{p}=0.002)$, meningiomas $(11 \%$ vs $0 \%, \mathrm{p}=0.009)$, and ependymomas ( $15 \%$ vs $2 \%, \mathrm{p}=0.009)$, the plastic surgery closure group included more chordomas $(20 \%$ vs $2 \%, \mathrm{p}=$ $0.005)$ and renal cell carcinoma metastases $(15 \%$ vs $0 \%$, $\mathrm{p}=0.006$ ). Tumor location did not differ significantly between groups, except that there was a higher proportion of sacral and coccygeal lesions in the plastic surgery closure group $(25 \%$ vs $7 \%, p=0.012)$, likely reflecting greater difficulty in wound closure and a perceived increased risk of wound infection for lesions closer to the anus.

\section{Operative and Postoperative Characteristics}

The plastic surgery and neurosurgery groups also differed significantly in terms of key neurosurgical details. Patients in the plastic surgery closure group were more likely to undergo a spondylectomy or corpectomy procedure as part of the index surgery ( $45 \%$ vs $20 \%, \mathrm{p}=0.006)$, and were more likely to have posterior fixation hardware placed $(75 \%$ vs $52 \%, \mathrm{p}=0.014)$ and significantly longer incisions (mean $7.2 \pm 3.6$ vs $4.5 \pm 2.6$ spinal levels, $\mathrm{p}<$ 0.001 ). Patients in the plastic surgery closure group were also more likely to receive demineralized bone matrix $(55 \%$ vs $33 \%, \mathrm{p}=0.022)$. Although all patients received intravenous infection prophylaxis, the use of intrawound vancomycin powder was significantly higher in the plastic surgery closure group $(77 \%$ vs $46 \%, p<0.001)$. Average total surgical procedure time was greater in the plastic surgery group ( $413 \pm 161$ vs $301 \pm 181$ minutes, $\mathrm{p}=0.001)$ and involved significantly more blood loss (906 \pm 1106 vs $283 \pm 373 \mathrm{ml}, \mathrm{p}<0.001)$. Taken together, these differences support the hypothesis that the procedures undergone by patients who received complex plastic surgery closure were more technically complex, even independent of other risk factors.

Additional operative and postoperative details for both groups are represented in Table 3 , along with a breakdown of flap type for the 60 patients in the complex plastic surgery closure group. The majority underwent mobilization and local advancement of paraspinous muscle flaps (63\%). The mean hospital length of stay before discharge was 9.2 \pm 8.1 days in the plastic surgery closure group, and $6.8 \pm$ 6.5 days in the neurosurgery closure group $(p=0.106)$. The combined mean follow-up visit period was 13.1 months (range 0.4-48.9 months) and was not significantly different between the neurosurgery and plastic surgery groups (12.0 vs 14.1 months, $p=0.383$ ). A total of 14 patients (5 in the neurosurgery group and 9 in the plastic surgery group, $p=0.538$ ) did not have office visits documented after discharge from the index operation.

\section{Risk Distribution and Wound Outcomes}

Using the categorization schema presented in Table 1, the number of preoperative risk factor categories present for each patient was computed. As seen in Fig. 2, the distribution of the number of risk categories across each group is approximately normal. The average number of risk factor categories present in patients from the plastic surgery closure group was 2.57 , as compared to 1.74 in the neurosurgery closure group. This difference between groups was highly significant $(\mathrm{p}<0.001)$, suggesting higher preoperative risk in the plastic surgery group.

Despite higher approximated risk for poor wound outcome, the plastic surgery group did not show an expected higher frequency of wound complications in any of the predefined categories, or across all wound complications (Table 4). The overall rate of wound complications, i.e., the number of patients who experienced at least one wound complication, was $24 \%$ (28\% in the plastic surgery group vs $17 \%$ in the neurosurgery group, $\mathrm{p}=0.145$ ). The plastic surgery group did have a higher rate of SSI (12\% vs $9 \%$, $\mathrm{p}=0.623)$ and seroma (17\% vs $7 \%, \mathrm{p}=0.117)$, although these differences were not statistically significant. Conversely, the neurosurgery closure group had slightly higher rates of wound dehiscence ( $13 \%$ vs $12 \%, \mathrm{p}=0.832$ ), hematoma ( $7 \%$ vs $5 \%, \mathrm{p}=0.719)$, and CSF leak (7\% vs $2 \%, \mathrm{p}=$ $0.204)$. The rates of skin necrosis (1 patient in each group) were approximately equal $(1.7 \%$ vs $2.2 \%, \mathrm{p}=0.851)$. Differences in rate of reoperation (17\% in the plastic surgery group vs $9 \%$ in the neurosurgery group, $\mathrm{p}=0.234$ ) and allcause mortality at the time of last follow-up (30\% in the plastic surgery group vs $15 \%$ in the neurosurgery group, $\mathrm{p}=0.076$ ) were also not significantly different between groups. Of 25 patients who died, 1 patient in each group died of infection related to wound complications. All other deaths were attributed to treatment-resistant systemic metastatic disease or other medical events following surgery unrelated to the surgical wound.

Results from regression analyses are represented in Table 5. Across the full patient cohort (pooling both groups), all individual risk factors were associated with several of the wound outcome variables tested. Factors associated with the most (5 or more) wound complications included smoking, diabetes, hypertension, preoperative chemotherapy or radiation, high preoperative BMI, size of wound, and number of levels instrumented. Diabetes, high BMI, and multilevel instrumentation were all associated via multivariate regression with multiple wound complications. This finding is consistent with other studies and makes sense in light of leading hypotheses regarding the association of obesity and impaired glucose metabolism with reduced lymphocytic defense against surgical pathogens. ${ }^{4,10}$ Overall, skin necrosis and CSF leak were the complications associated with the fewest risk factors. These complications were also observed with the lowest frequency across our data. Furthermore, univariate regression of the number of risk factor categories with each outcome showed significant associations $(\mathrm{p}<0.05)$ with all wound outcomes tested. As expected, the number of risk factor categories was not significantly associated with outcome by multivariate regression because this composite variable is a linear combination of other dependent risk variables. These results indicate that the number of risk factor categories present was a robust predictor of outcome.

\section{Discussion}

This study supports the hypothesis that plastic surgery 
TABLE 3. Operative and postoperative details for full patient cohort, as well as for complex (plastic surgery) and simple (neurosurgery) wound closure subpopulations

\begin{tabular}{|c|c|c|c|c|}
\hline Operative \& Postop Details & Total Cohort $(n=106)$ & Complex Closure Group $(n=60)$ & Simple Closure Group $(n=46)$ & p Value \\
\hline \multicolumn{5}{|l|}{ Procedures performed, n (\%) } \\
\hline Laminectomy/laminotomy & $91(86)$ & $49(82)$ & $42(91)$ & 0.161 \\
\hline Facetectomy/foraminotomy & $13(12)$ & $7(12)$ & $6(13)$ & 0.832 \\
\hline Fusion/arthrodesis & $53(50)$ & $34(57)$ & $19(41)$ & 0.119 \\
\hline Spondylectomy/corpectomy & $36(34)$ & $27(45)$ & $9(20)$ & 0.006 \\
\hline \multicolumn{5}{|l|}{ Wound size $($ mean $\pm \mathrm{SD})$} \\
\hline No. of levels spanned by lesion & $2.4 \pm 1.7$ & $2.4 \pm 1.8$ & $2.4 \pm 1.6$ & 0.851 \\
\hline No. of levels incised & $6.1 \pm 3.5$ & $7.2 \pm 3.6$ & $4.5 \pm 2.6$ & $<0.001$ \\
\hline Length of closure, $\mathrm{cm}$ & - & $22.6 \pm 7.1$ & Not reported & - \\
\hline No. of levels instrumented & $4.1 \pm 3.7$ & $5.1 \pm 3.8$ & $2.9 \pm 3.2$ & 0.002 \\
\hline \multicolumn{5}{|l|}{ Instrumentation \& materials, n (\%) } \\
\hline Placement of any instrumentation & $69(65)$ & $45(75)$ & $24(52)$ & 0.014 \\
\hline Placement of screws \& rods & $60(57)$ & $42(70)$ & $18(39)$ & 0.001 \\
\hline Placement of screws \& plates & $16(15)$ & $7(12)$ & $9(20)$ & 0.264 \\
\hline Placement of expandable cage & $28(26)$ & $18(30)$ & $10(22)$ & 0.343 \\
\hline Use of demineralized bone matrix & $48(45)$ & $33(55)$ & $15(33)$ & 0.022 \\
\hline \multicolumn{5}{|l|}{ Antibiotics, $n(\%)$} \\
\hline Use of vancomycin powder & $67(63)$ & $46(77)$ & $21(46)$ & $<0.001$ \\
\hline Use of vancomycin nonpowder & $73(69)$ & $49(82)$ & $24(52)$ & $<0.001$ \\
\hline Use of other antibiotic infusion & $106(100)$ & $60(100)$ & $46(100)$ & - \\
\hline \multicolumn{5}{|l|}{ Closure technique, $\mathrm{n}(\%)$} \\
\hline Simple layered closure & - & - & $46(100)$ & - \\
\hline Closure w/ paraspinous flaps & - & $38(63)$ & - & - \\
\hline Closure w/ trapezius flaps & - & $8(13)$ & - & - \\
\hline Closure w/ latissimus flaps & - & $3(5)$ & - & - \\
\hline Closure w/ gluteal flaps & - & $6(10)$ & - & - \\
\hline Closure w/ rotational flap & - & $1(2)$ & - & - \\
\hline Closure w/ V-Y flap & - & $1(2)$ & - & - \\
\hline Closure w/ local advancement flap NOS & - & $3(5)$ & - & - \\
\hline \multicolumn{5}{|l|}{ Other intraop details (mean \pm SD) } \\
\hline Procedure duration, min & $364 \pm 178$ & $413 \pm 161$ & $301 \pm 181$ & 0.001 \\
\hline Estimated blood loss, $\mathrm{ml}$ & $635 \pm 918$ & $906 \pm 1106$ & $283 \pm 373$ & $<0.001$ \\
\hline \multicolumn{5}{|l|}{ Postop course } \\
\hline Mean hospital stay duration $\pm S D$, days & $8.1 \pm 7.5$ & $9.2 \pm 8.1$ & $6.8 \pm 6.5$ & 0.106 \\
\hline Mean follow-up period $\pm \mathrm{SD}$, mos & $13.1 \pm 11.7$ & $14.1 \pm 12.7$ & $12.0 \pm 12.3$ & 0.383 \\
\hline Postop chemotherapy, n (\%) & $28(26)$ & $19(32)$ & $9(20)$ & 0.135 \\
\hline Postop radiation, $\mathrm{n}(\%)$ & $43(41)$ & $31(52)$ & $12(26)$ & 0.006 \\
\hline
\end{tabular}

NOS $=$ not otherwise specified.

Continuous variables are reported as averages with standard deviations. The $p$ values represent results of unpaired t-tests of the difference between the two subpopulations with respect to the variable indicated. Boldface type indicates statistical significance.

involvement in the primary closure of complex wounds following resection of spinal tumors is beneficial, especially among the highest-risk patients. A limited number of reports from other comprehensive spinal oncology centers have documented similar findings regarding wound complication rates. The largest series of wound closures by plastic surgery, reported by a group from Johns Hopkins (Carl et al. 2017), retrospectively reviewed the charts of 288 patients over a 12-year period and published a reoper- ation rate of $27 \%$, infection rate of $24 \%$, wound dehiscence rate of $17 \%$, and CSF leak rate of $7 \% .{ }^{6}$ Our complex plastic surgery closure population's complication rates were comparable or lower, despite the fact that the study population of Carl et al. included fewer patients with metastatic lesions (22.6\%) and had lower baseline rates of multiple major wound complication risk factors $(12.2 \%$ chronic steroids, $35.3 \%$ preoperative radiation, $23.6 \%$ chemotherapy, $12.8 \%$ smoking). Another recent study of 55 patients by a 


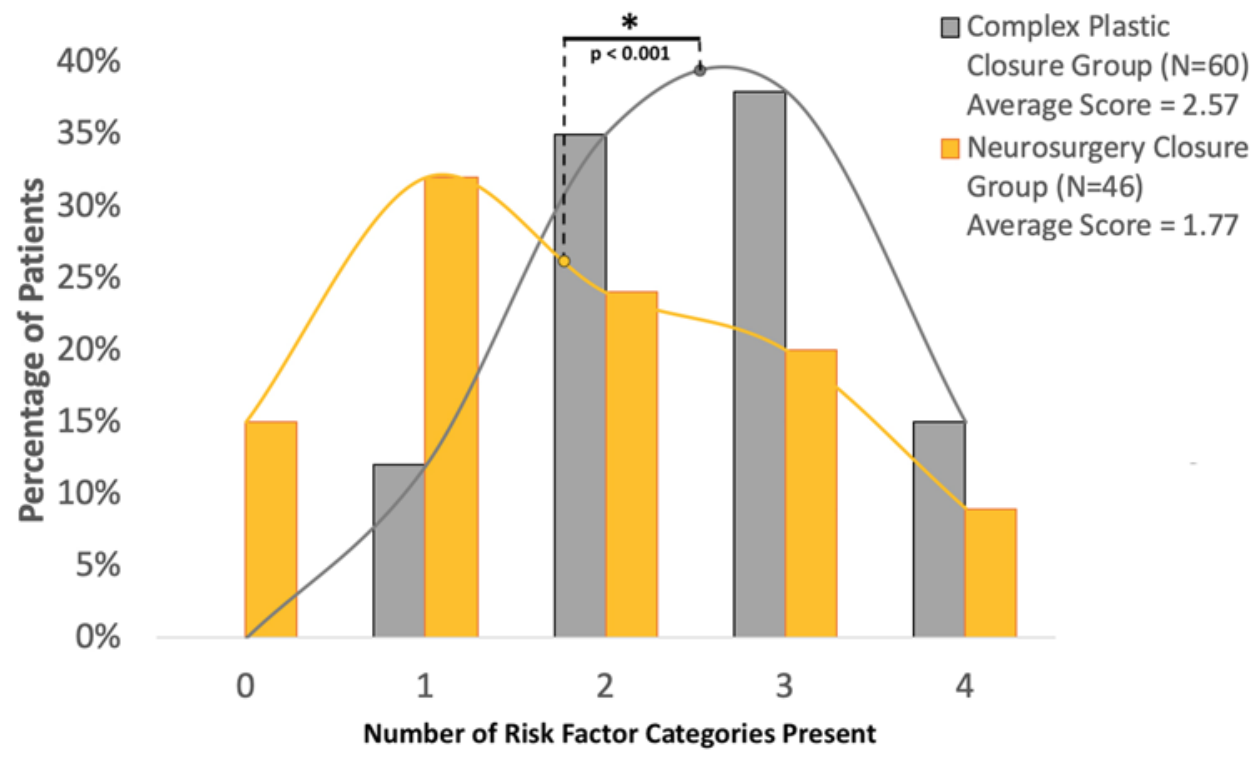

FIG. 2. Bar graph representing the number of risk categories present among patients in the complex plastic closure $(n=60)$ and neurosurgery closure $(n=46)$ groups. Bars represent percentage of patients in each group in which each number of risk factor categories is present. Continuous lines represent approximate distribution of number of risk categories in each group, with averages indicated by points (2.57 risk categories present in the plastic surgery group and 1.77 risk categories present in the neurosurgery group). The difference in average number of risk categories present in each group is statistically significant $\left({ }^{*} p<0.001\right)$. Figure is available in color online only.

group at Cornell (Franck et al. 2018) reported even lower infection rates $(1.7 \%)$ than our study, but similar rates of reoperation $(11.7 \%)$ and seroma $(15 \%)$ in an oncological population. ${ }^{13}$ This study population also had lower baseline rates of some particularly relevant wound complication risk factors (13\% diabetes, 30\% smoking history). It is not unexpected that rates will vary somewhat with practice group and institution.

Notably, we are not aware of any previous studies that have directly compared wound outcomes between patient cohorts who did or did not undergo complex plastic closure for neoplastic disease. This gap in the literature may be due, in part, to bias between groups in terms of underlying baseline wound complication risk, a potentially important confounding variable that, unless clearly quantified and reported, would make the results of such a study difficult to interpret. Furthermore, we found no validated risk scoring systems previously published that stratify baseline risk for wound complication arising from multiple sources in the spinal oncology population. To solve this problem in the present work, we accounted for consensus factors identified by the spine surgery and spinal oncology literature as likely contributing to higher rates of wound complications in this population, and pooled factors into a categorical composite measure counting the number of risk categories (out of four) that were present before surgery. We also compared the prevalence of individual risk factors between groups.

By quantifying risk in this way, we were able to characterize the risk bias between our two cohorts, and thereby better contextualize differences in outcome between the two. While there were not significant differences between populations in terms of rate of any wound outcome docu- mented in our study, there was a highly significant difference in number of risk factor categories present (1.77 in the neurosurgery group vs 2.57 in the plastic surgery group, $\mathrm{p}<0.001$ ), indicating that patients in the plastic surgery group had more types of relevant risk factors than patients in the neurosurgery group. Accordingly, we report retrospective evidence that having plastic surgery involvement in wound closure may reduce wound complication rates in spinal oncology patients with high baseline risk. While this trend, observed in a multipractitioner data set, is not likely attributable to difference in skill between practitioners of neurological or plastic surgery, it may suggest the importance of muscle flaps in improving wound complication profiles among this population. This makes sense given the relevant background literature on musculocutaneous flap utilization in spinal wound reapproximation..$^{5-9,12-14,22}$ Moreover, our study suggests that patients with complex spinal oncology may be better treated in a multidisciplinary environment that capitalizes on the specific, unique expertise of each surgical group involved. The criteria to be used for determining need for multidisciplinary surgical care on a case-by-case basis are yet to be standardized.

Quantifying preoperative risk categorically as we have done in this study may hold particular utility for spinal oncology, as this strategy incorporates medical history, surgical planning, and physiological factors that are common and easily identifiable in the preoperative setting for this population. Grouping factors categorically ensures that no single class of factors has a disproportionate weight on the risk approximation, even in the presence of multiple positive factors within a given category. Furthermore, our regression analyses suggest that number of risk factor cat- 
TABLE 4. Composite risk rating, wound complication rates, and outcomes for the full patient cohort, as well as for complex (plastic surgery) and simple (neurosurgery) wound closure subpopulations

\begin{tabular}{|c|c|c|c|c|}
\hline $\begin{array}{c}\text { No. of Risk Factor Categories \& Outcome } \\
\text { Distributions }\end{array}$ & Total Cohort $(n=106)$ & Complex Closure Group $(n=60)$ & Simple Closure Group $(n=46)$ & $\mathrm{p}$ Value \\
\hline \multicolumn{5}{|l|}{ No. of risk factor categories, $n(\%)$} \\
\hline 0 & $7(7)$ & $0(0)$ & $7(15)$ & - \\
\hline 1 & $22(21)$ & $7(12)$ & $15(33)$ & - \\
\hline 2 & $32(30)$ & $21(35)$ & $11(24)$ & - \\
\hline 3 & $32(30)$ & $23(38)$ & $9(20)$ & - \\
\hline 4 & $13(12)$ & $9(15)$ & $4(9)$ & - \\
\hline Average & 2.21 & 2.57 & 1.74 & $<0.001$ \\
\hline \multicolumn{5}{|l|}{ Wound complication rates \& outcome, $n(\%)$} \\
\hline Any wound complication & $25(24)$ & $17(28)$ & $8(17)$ & 0.145 \\
\hline SSI & $11(10)$ & $7(12)$ & $4(9)$ & 0.623 \\
\hline Skin necrosis & $2(2)$ & $1(2)$ & $1(2)$ & 0.851 \\
\hline Dehiscence & $13(12)$ & $7(12)$ & $6(13)$ & 0.832 \\
\hline Hematoma & $6(6)$ & $3(5)$ & $3(7)$ & 0.719 \\
\hline Seroma & $13(12)$ & $10(17)$ & $3(7)$ & 0.117 \\
\hline CSF leak & $3(3)$ & $1(2)$ & $3(7)$ & 0.204 \\
\hline Need for reoperation & $14(13)$ & $10(17)$ & $4(9)$ & 0.234 \\
\hline Mortality (all cause) & $18(17)$ & $18(30)$ & $7(15)$ & 0.076 \\
\hline Mortality (wound-related) & $2(2)$ & $1(2)$ & $1(2)$ & 0.851 \\
\hline
\end{tabular}

Results of unpaired t-tests for significance of difference between the two subpopulations found to be highly significant $(p<0.001)$ with respect to risk rating, but not with respect to any of the measured wound complication rates. Boldface type indicates statistical significance.

egories present may be a measure particularly well tuned to estimating risk of wound complication in the spinal oncology population. Were data like those presented in this study used to develop a risk scoring system based on these principles, such a system could be easily implementable as a useful clinical tool. However, future studies should more thoroughly test for associations between these variables (as well as other variables not tested in this study but reported in the broader spine surgery literature) and wound outcomes. ${ }^{1,3,4,16,18,19,23,29}$ All risk variables tested in this study were reported in the spinal oncology literature previously, but comparison of their relative associations with wound outcomes was limited here by modest sample size $(n=106$ overall, $n=46$ vs $n=60$ in each cohort) and low incidence of individual complications. Furthermore, in these data, there were few patients positive for all four risk categories (15\% in the plastic surgery group, $9 \%$ in the neurosurgery group), possibly due to a subset of the highest-risk patients having been excluded from surgery, and therefore excluded from this study. Building on the results of our analyses in future prospective and larger retrospective studies will be essential for developing useful prognostication systems. Such tools could be especially useful for subpopulations of particular interest within neurosurgical oncology, such as patients preoperatively treated with chemotherapy or radiation.

In this study, we focused on quantifying preoperative risk as a means of standardizing groups for comparison of outcomes. However, limitations of our retrospective study include lack of control for technical variability between multiple practitioners as well as possible sources of selection bias. Our data suggest that there may be bias between groups in terms of specific measures taken to prevent wound infection when treating patients perceived to be at high risk. For example, while intraoperative antibiotics were administered to all patients (100\%) across both groups, patients in the plastic surgery group were significantly more likely to get intraoperative vancomycin, either as a wound powder (77\% vs $46 \%, \mathrm{p}<0.001)$ or in other forms ( $82 \%$ vs $52 \%, \mathrm{p}<0.001)$. Furthermore, cases in the neurosurgery group were typically performed using simple layered closure, while plastic surgery practitioners used musculocutaneous flap techniques for closure, and the majority of cases in which wound drains were placed also involved plastic surgery. These differences in wound care observed between groups may highlight more granular differences in practice that could be individually assessed in future studies as the underlying causes of better outcomes in the plastic surgery group; the present study, however, considers them as collectively attributable to the composition of the surgical team. Another source of selection bias may include the level of training of surgical practitioners closing wounds in each group. As in any academic institution, including our own, a surgical trainee (i.e., resident or fellow) almost always participates in wound closures by both neurosurgery and plastic surgery, making it difficult to differentiate groups. However, an attending physician is always present for closure in either group. In general, the effect of surgical practitioner level of training on wound outcomes in spinal surgery represents another avenue of future investigation.

In light of data collected during this study, such con- 
TABLE 5. Results of univariate and multivariate linear regression models assessing association between individual outcome variables (wound complications, reoperation, mortality) with individually identified risk factors and the composite number of risk factor categories present across the full patient cohort $(n=106)$

\begin{tabular}{|c|c|c|c|c|c|c|c|c|c|}
\hline Risk Factor & SSI & Skin Necrosis & Dehiscence & Hematoma & Seroma & CSF Leak & Reoperation & Mortality & Any Wound Complication \\
\hline Smoking & U & - & U & U & U & - & U & U & U \\
\hline Diabetes mellitus & M & U & $M$ & U & - & $U$ & M & M & $M$ \\
\hline Hypertension & U & - & U & U & $U$ & - & U & U & U \\
\hline Anticoagulation & U & - & U & - & - & - & U & $U$ & $U$ \\
\hline Chemotherapy & U & - & U & $U$ & $U$ & - & U & $U$ & $U$ \\
\hline Radiation & U & - & U & U & U & - & U & U & $U$ \\
\hline Preop BMI & U & - & U & $U$ & M & $U$ & U & M & U \\
\hline Prior spine surgery & U & - & U & - & - & - & U & - & - \\
\hline Age & U & - & U & U & $U$ & - & U & $U$ & $U$ \\
\hline \multicolumn{10}{|l|}{ No. of levels } \\
\hline Incised & U & - & U & $U$ & $U$ & - & U & $U$ & $U$ \\
\hline Tumor location & U & - & $U$ & $U$ & $U$ & - & U & $U$ & U \\
\hline Instrumented & $U$ & - & M & - & M & $U$ & U & M & $U$ \\
\hline Duration of procedure & U & - & M & - & $U$ & - & $U$ & U & $U$ \\
\hline Estimated blood loss & U & - & $U$ & - & - & - & $U$ & $U$ & $U$ \\
\hline No. of risk categories & $U$ & $U$ & $U$ & $U$ & $U$ & $U$ & $U$ & $U$ & $U$ \\
\hline
\end{tabular}

$M=$ significance $(p<0.05)$ of association by multivariate regression; $U=$ significance $(p<0.05)$ of association by univariate regression; $-=$ nonsignificance by both univariate and multivariate regression.

founding biases between neurosurgical and plastic surgery care teams with respect to wound care speak more to the need for greater standardization in wound complication prophylaxis than to the efficacy of individual surgical techniques. However, by showing through post hoc regression analyses that the risk variables studied were associated with wound outcome in the same cohort of patients, we demonstrated that these potential biases probably had a limited effect on our results. Overall, stratifying risk using a composite scoring system specifically catered to the spinal oncology population may be a reasonable approach to prognosticating for these patients, and specifically for deciding whether a more sophisticated wound closure technique performed by a multidisciplinary surgical team may be the superior wound closure option for individual cases. Such complex wound care may be especially important for yet unidentified subgroups of patients, like those exposed to longer hospital stays and prolonged periods of stasis.

\section{Conclusions}

To our knowledge, the present study is the first to compare wound outcomes specifically between patients who did or did not undergo complex plastic surgery closure of their posterior spine wound following spine tumor resection. By stratifying preoperative risk for this implicitly high-risk population, we were able to document the bias between cohorts in terms of likelihood of complication and demonstrate the mitigation of this risk by the complex plastic surgery closure strategy. At other spinal oncology centers with similar resources, complex plastic surgery wound closure via flap mobilization should be considered in patients with neoplastic spinal disease and multiple types of baseline risk factors. While we provide strong preliminary evidence that the identified risk factors and categorical stratification presented are indeed associated with wound complication, these data should be further validated in future studies in order to derive a risk scoring system for wound outcome prediction for this population.

\section{Acknowledgments}

We would like to acknowledge Kendall Rivera-Lane, medical illustrator for the Rhode Island Hospital Department of Neurosurgery, for contributing photography of our illustrative case for this paper, and Steven E. Reinert, informaticist at Lifespan Health System, for help in obtaining the list of patients meeting inclusion criteria for this study.

\section{References}

1. Abdul-Jabbar A, Takemoto S, Weber MH, Hu SS, Mummaneni PV, Deviren V, et al: Surgical site infection in spinal surgery: description of surgical and patient-based risk factors for postoperative infection using administrative claims data. Spine (Phila Pa 1976) 37:1340-1345, 2012

2. Atesok K, Papavassiliou E, Heffernan MJ, Tunmire D, Sitnikov I, Tanaka N, et al: Current strategies in prevention of postoperative infections in spine surgery. Global Spine J [epub ahead of print], 2019

3. Bekelis K, Desai A, Bakhoum SF, Missios S: A predictive model of complications after spine surgery: the National Surgical Quality Improvement Program (NSQIP) 2005-2010. Spine J 14:1247-1255, 2014

4. Brem H, Tomic-Canic M: Cellular and molecular basis of wound healing in diabetes. J Clin Invest 117:1219-1222, 2007

5. Calderon W, Chang N, Mathes SJ: Comparison of the effect of bacterial inoculation in musculocutaneous and fasciocutaneous flaps. Plast Reconstr Surg 77:785-794, 1986

6. Carl HM, Coon D, Calotta NA, Pedreira R, Sacks JM: Surgi- 
cal factors associated with prolonged hospitalization after reconstruction for oncological spine surgery. Plast Reconstr Surg Glob Open 5:e1271, 2017

7. Chang DW, Friel MT, Youssef AA: Reconstructive strategies in soft tissue reconstruction after resection of spinal neoplasms. Spine (Phila Pa 1976) 32:1101-1106, 2007

8. Chieng LO, Hubbard Z, Salgado CJ, Levi AD, Chim H: Reconstruction of open wounds as a complication of spinal surgery with flaps: a systematic review. Neurosurg Focus 39(4): E17, 2015

9. Cohen LE, Fullerton N, Mundy LR, Weinstein AL, Fu KM, Ketner JJ, et al: Optimizing successful outcomes in complex spine reconstruction using local muscle flaps. Plast Reconstr Surg 137:295-301, 2016

10. Doyle SL, Lysaght J, Reynolds JV: Obesity and post-operative complications in patients undergoing non-bariatric surgery. Obes Rev 11:875-886, 2010

11. Dumanian GA, Ondra SL, Liu J, Schafer MF, Chao JD: Muscle flap salvage of spine wounds with soft tissue defects or infection. Spine (Phila Pa 1976) 28:1203-1211, 2003

12. Eshima I, Mathes SJ, Paty P: Comparison of the intracellular bacterial killing activity of leukocytes in musculocutaneous and random-pattern flaps. Plast Reconstr Surg 86:541-547, 1990

13. Franck P, Bernstein JL, Cohen LE, Härtl R, Baaj AA, Spector JA: Local muscle flaps minimize post-operative wound morbidity in patients with neoplastic disease of the spine. Clin Neurol Neurosurg 171:100-105, 2018

14. Garvey PB, Rhines LD, Dong W, Chang DW: Immediate soft-tissue reconstruction for complex defects of the spine following surgery for spinal neoplasms. Plast Reconstr Surg 125:1460-1466, 2010

15. Hultman CS, Jones GE, Losken A, Seify H, Schaefer TG, Zapiach LA, et al: Salvage of infected spinal hardware with paraspinous muscle flaps: anatomic considerations with clinical correlation. Ann Plast Surg 57:521-528, 2006

16. Janssen DMC, van Kuijk SMJ, d'Aumerie B, Willems P: A prediction model of surgical site infection after instrumented thoracolumbar spine surgery in adults. Eur Spine J 28:775782, 2019

17. Klink BK, Thurman RT, Wittpenn GP, Lauerman WC, Cain JE: Muscle flap closure for salvage of complex back wounds. Spine (Phila Pa 1976) 19:1467-1470, 1994

18. Lai Q, Song Q, Guo R, Bi H, Liu X, Yu X, et al: Risk factors for acute surgical site infections after lumbar surgery: a retrospective study. J Orthop Surg Res 12:116, 2017

19. Martin CT, Pugely AJ, Gao Y, Ilgenfritz RM, Weinstein SL: Incidence and risk factors for early wound complications after spinal arthrodesis in children: analysis of 30-day follow-up data from the ACS-NSQIP. Spine (Phila Pa 1976) 39:1463-1470, 2014

20. Meredith DS, Kepler CK, Huang RC, Brause BD, BoachieAdjei O: Postoperative infections of the lumbar spine: presentation and management. Int Orthop 36:439-444, 2012

21. Mericli AF, Tarola NA, Moore JH Jr, Copit SE, Fox JW IV, Tuma GA: Paraspinous muscle flap reconstruction of complex midline back wounds: risk factors and postreconstruction complications. Ann Plast Surg 65:219-224, 2010
22. Murphy RC, Robson MC, Heggers JP, Kadowaki M: The effect of microbial contamination on musculocutaneous and random flaps. J Surg Res 41:75-80, 1986

23. Nahhas CR, Hu K, Mehta AI: Incidence and risk factors of wound complications in long segment instrumented thoracolumbar spinal fusions: a retrospective study. J Spine Surg 4:233-240, 2018

24. Omeis IA, Dhir M, Sciubba DM, Gottfried ON, McGirt MJ, Attenello FJ, et al: Postoperative surgical site infections in patients undergoing spinal tumor surgery: incidence and risk factors. Spine (Phila Pa 1976) 36:1410-1419, 2011

25. Sambri A, Gasbarrini A, Cialdella S, De Iaco P, Boriani S: Pedicled omental flaps in the treatment of complex spinal wounds after en bloc resection of spine tumors. J Plast Reconstr Aesthet Surg 70:1267-1271, 2017

26. Sundaresan N, Rothman A, Manhart K, Kelliher K: Surgery for solitary metastases of the spine: rationale and results of treatment. Spine (Phila Pa 1976) 27:1802-1806, 2002

27. Vitaz TW, Oishi M, Welch WC, Gerszten PC, Disa JJ, Bilsky $\mathrm{MH}$ : Rotational and transpositional flaps for the treatment of spinal wound dehiscence and infections in patient populations with degenerative and oncological disease. J Neurosurg 100 (1 Suppl Spine):46-51, 2004

28. Wise JJ, Fischgrund JS, Herkowitz HN, Montgomery D, Kurz LT: Complication, survival rates, and risk factors of surgery for metastatic disease of the spine. Spine (Phila Pa 1976) 24:1943-1951, 1999

29. Xing D, Ma JX, Ma XL, Song DH, Wang J, Chen Y, et al: A methodological, systematic review of evidence-based independent risk factors for surgical site infections after spinal surgery. Eur Spine J 22:605-615, 2013

\section{Disclosures}

Dr. Oyelese reports being a consultant for Brainlab.

\section{Author Contributions}

Conception and design: Leary, Boyajian, Syed, Oyelese, Woo, Gokaslan, Fridley. Acquisition of data: Leary, DD Liu, Boyajian, Crozier. Analysis and interpretation of data: Leary, DD Liu, Syed, Crozier, Oyelese, Woo, Gokaslan, Fridley. Drafting the article: Leary, DD Liu, Fridley. Critically revising the article: Leary, DD Liu, Boyajian, Syed, Camara-Quintana, Niu, Svokos, Oyelese, PY Liu, Woo, Gokaslan, Fridley. Reviewed submitted version of manuscript: Leary, Syed, Woo, Gokaslan, Fridley. Approved the final version of the manuscript on behalf of all authors: Leary. Statistical analysis: Leary, DD Liu. Administrative/technical/ material support: Camara-Quintana, Niu, Svokos, Crozier, Oyelese, PY Liu, Woo, Gokaslan, Fridley. Study supervision: Syed, Camara-Quintana, Niu, Svokos, Oyelese, PY Liu, Woo, Gokaslan, Fridley.

\section{Correspondence}

Owen P. Leary: Warren Alpert Medical School of Brown University and Rhode Island Hospital, Providence, RI. owen leary@brown.edu. 